\title{
Photoluminescence quantum efficiency of dense silicon nanocrystal ensembles in $\mathrm{SiO}_{2}$
}

\author{
R. J. Walters, ${ }^{1}$ J. Kalkman, ${ }^{2}$ A. Polman, ${ }^{2}$ H. A. Atwater, ${ }^{1}$ and M. J. A. de Dood ${ }^{3}$ \\ ${ }^{1}$ Thomas J. Watson Laboratory of Applied Physics, California Institute of Technology, Pasadena, California 91125, USA \\ ${ }^{2}$ Center for Nanophotonics, FOM Institute for Atomic and Molecular Physics, Kruislaan 407, 1098 SJ Amsterdam, The Netherlands \\ ${ }^{3}$ Leiden Institute of Physics, University of Leiden, Niels Bohrweg 2, 2333 CA Leiden, The Netherlands \\ (Received 13 September 2005; revised manuscript received 27 February 2006; published 28 April 2006)
}

\begin{abstract}
The photoluminescence decay characteristics of silicon nanocrystals in dense ensembles fabricated by ion implantation into silicon dioxide are observed to vary in proportion to the calculated local density of optical states. A comparison of the experimental $1 / e$ photoluminescence decay rates to the expected spontaneous emission rate modification yields values for the internal quantum efficiency and the intrinsic radiative decay rate of silicon nanocrystals. A photoluminescence quantum efficiency as high as $59 \% \pm 9 \%$ is found for nanocrystals emitting at $750 \mathrm{~nm}$ at low excitation power. A power dependent nonradiative decay mechanism reduces the quantum efficiency at high pump intensity.
\end{abstract}

DOI: 10.1103/PhysRevB.73.132302

PACS number(s): 78.67.Bf, 78.55.-m

Since Canham's first report of bright, room-temperature luminescence from porous silicon, ${ }^{1}$ nanostructured silicon has been regarded as a promising materials system for silicon-based optoelectronic technologies. ${ }^{2}$ It is now widely accepted that near infrared photoluminescence in silicon nanocrystals originates from the band-to-band recombination of quantum confined excitons. ${ }^{3}$ This emission is much more efficient than the corresponding process in bulk silicon because the luminescing nanocrystals are substantially free of impurities and lattice defects that can lead to nonradiative recombination. Quantum confinement in nanocrystals additionally offers the advantages of a size-tunable electronic band gap and an improved oscillator strength for radiative recombination. Despite technological challenges in electrically pumping quantum dots, there is optimism that silicon nanocrystals may someday be part of a device technology for light emission that competes favorably with III-V semiconductors. This optimism is buoyed by ever increasing economies of scale for silicon processing and reports of high quantum efficiency in single nanocrystals., ${ }^{4,5}$ However, quantitative values for the radiative quantum efficiency of silicon nanocrystals in dense, devicelike ensembles have not been reported to date.

In this report we present measurements of the internal quantum efficiency of silicon nanocrystals similar to those employed in electroluminescent devices. ${ }^{6}$ The basis of the experiment is the modification of the spontaneous emission rate of a dipole emitter in proportion to the local density of optical states (LDOS). ${ }^{7-11}$ In contrast to commonly used methods that measure the ratio of output power to input power, this technique does not require the use of a reference sample or the estimation of excitation or collection efficiency. A similar approach has previously been applied to silicon nanocrystal ensembles in close proximity to $\mathrm{Ag}$ films. ${ }^{12}$ Our samples consist of identical ensembles of silicon nanocrystals embedded in oxide layers of differing thickness on a silicon substrate. The photoluminescence (PL) decay rate of the nanocrystals varies with the changing LDOS as the separation distance between the ensemble and the silicon substrate is altered. The quantum efficiency and the decay rates are found by comparing the magnitude of the decay rate modification to a calculation of the LDOS. This approach allows rates to be independently determined for both the radiative and the nonradiative decay components. We also identify a power-dependent component of the measured nanocrystal decay rate, which is indicative of complex photocarrier dynamics and interactions among nanocrystals under intense excitation.

Two samples were prepared via computer controlled immersion of $\mathrm{a} \mathrm{SiO}_{2}$ layer into a buffered HF solution (see Fig. 1 inset). Sample $A$ was fabricated from an initially $1 \mu \mathrm{m}$ thick $\mathrm{SiO}_{2}$ layer grown by wet thermal oxidation at an etch rate of $1 \mathrm{~nm} / \mathrm{s}\left(1: 448 \% \mathrm{HF}: \mathrm{H}_{2} \mathrm{O}\right)$, while sample $B$ was etched at a rate of $0.25 \mathrm{~nm} / \mathrm{s}\left(1: 2048 \% \mathrm{HF}: \mathrm{H}_{2} \mathrm{O}\right)$ from an initially $105 \mathrm{~nm}$ thick oxide grown by dry oxidation. The resulting thickness profiles were measured using spatially resolved spectral ellipsometry (shown for sample $A$ in Fig. 1). Both samples were implanted with ${ }^{28} \mathrm{Si}^{+}$ions at $5 \mathrm{keV}$ to a fluence of $1.2 \times 10^{16} \mathrm{ions} / \mathrm{cm}^{2}$. The resulting silicon depth profile, as obtained by a Monte-Carlo simulation using SRIM, ${ }^{13}$ peaks at a depth of $10 \mathrm{~nm}$ with a predicted 20 at. $\%$ excess Si concentration and $3 \mathrm{~nm}$ full width at half maximum. The two samples were annealed simultaneously in a tube furnace $\left(1100{ }^{\circ} \mathrm{C} ; 10 \mathrm{~min} ; 2000 \mathrm{ppm} \mathrm{O}\right.$ : Ar) in order to precipitate nanocrystals from the supersaturated solution. This process was followed by a second annealing step to passivate surface defects $\left(450{ }^{\circ} \mathrm{C} ; 30 \mathrm{~min} ; 10 \% \mathrm{H}_{2}: \mathrm{N}_{2}\right)$. This procedure forms $0.4-3.0 \times 10^{13}$ nanocrystals $/ \mathrm{cm}^{2}$ in an approximate monolayer near the peak of the implantation profile, with typical diameter $2-3 \mathrm{~nm} .{ }^{14}$

Silicon nanocrystal PL was measured at different positions along the sample at room temperature. The $488 \mathrm{~nm}$ line of an $\mathrm{Ar}^{+}$ion laser, focused to a $300 \mu \mathrm{m}$ diameter spot, was used to excite the nanocrystals at normal incidence. PL spectra were collected using a spectrograph equipped with a charge coupled device (CCD) array detector. A representative PL spectrum measured for nanocrystals embedded in a $105 \mathrm{~nm}$ thick oxide layer (sample $B$ ) shows broad nearinfrared emission (Fig. 1; inset), typical of silicon nanocrystal ensembles. A thermoelectrically cooled photomultiplier tube with photon counting electronics was used to collect time resolved data using an acousto-optic modulator to 


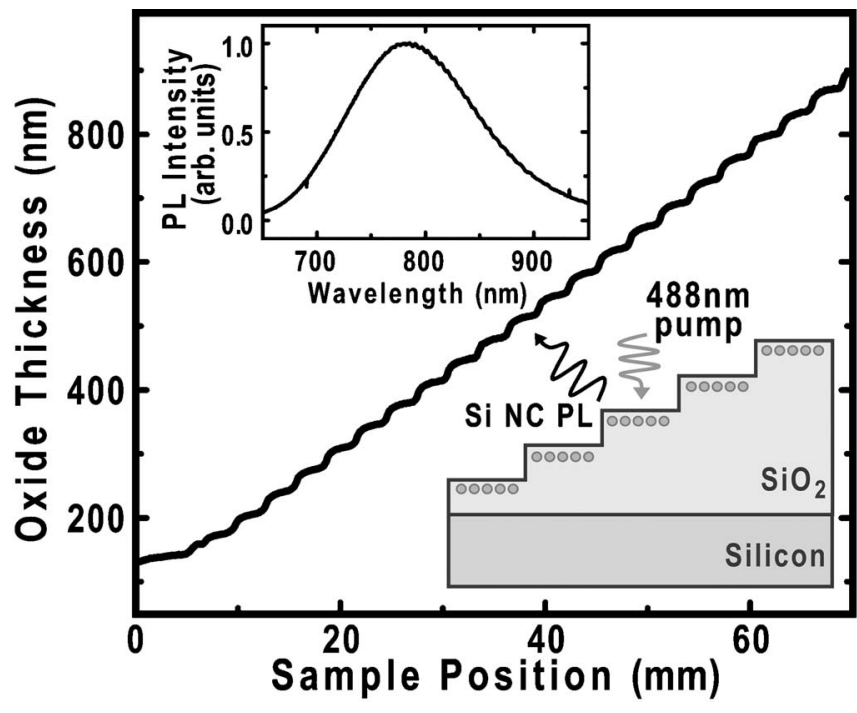

FIG. 1. Oxide thickness along a sample as measured with spectral ellipsometry. The silicon nanocrystals form near the $\mathrm{SiO}_{2}$-to-air interface as indicated schematically (inset, lower right). A representative nanocrystal PL spectrum shows typical near-infrared emission (inset, top left).

modulate the pump beam. Under high excitation power $\left(1 \mathrm{~W} / \mathrm{mm}^{2}\right)$, sufficient statistics were collected after a few minutes of signal integration. At low excitation power $\left(0.01-0.1 \mathrm{~W} / \mathrm{mm}^{2}\right)$, it was necessary to collect data for $1 \mathrm{~h}$ at each oxide thickness.

The decay of Si nanocrystals in a dense ensemble is commonly described by a stretched exponential or Kohlrausch decay of the form $I(t)=I_{0} \exp \left\{-\left(\Gamma_{K} t\right)^{\beta}\right\}$, where $\Gamma_{K}$ is the ensemble average $1 / e$ PL decay rate. ${ }^{15}$ The value of $\beta$ is typically near 0.7 for dense ensembles, while near singleexponential behavior $(\beta=1.0)$ is found in low-density ensembles. ${ }^{16,17}$ All our data are well fit using a fixed value of $\beta=0.7$. This suggests that energy transfer between neighboring nanocrystals contributes significantly to the observed decay rate in dense ensembles. Laplace transformation of the stretched exponential function with $\beta=0.7$ results in an underlying distribution of single exponential emitters that peaks at a decay rate of $0.435 \Gamma_{K}$. Unfortunately, the precise nature of the energy transfer process is still unknown and complicates the interpretation of $\Gamma_{K}$ as a measure of single exponential decay processes in the ensemble.

A model ${ }^{18}$ that assumes that an excited nanocrystal acts as a donor and transfers its energy to neighboring acceptor nanocrystals can be used to describe the Kohlrausch decay. This model takes the statistical average of the decay rate of the donors, using a distance dependent energy transfer rate $\eta(r)$. Assuming a uniform distribution of acceptors, the single exponential decay rate $\Gamma_{0}$ for an isolated donor can be calculated, yielding $\Gamma_{0}=0.357 \Gamma_{K}$ for dipole-dipole transfer $\left[\eta(r) \propto 1 / r^{6}\right]$ between nanocrystals and $\Gamma_{0}=0.314 \Gamma_{K}$ when a tunneling mechanism is assumed $[\eta(r) \propto \exp \{-\alpha r\}]$. A change in $\Gamma_{0}$ shifts the distribution in such a way that $\Gamma_{K}$ is always proportional to the intrinsic single exponential decay rate $\Gamma_{0}$. Consequently the relative change in $\Gamma_{K}$ is equal to the relative change in $\Gamma_{0}$, regardless of the transfer mechanism.

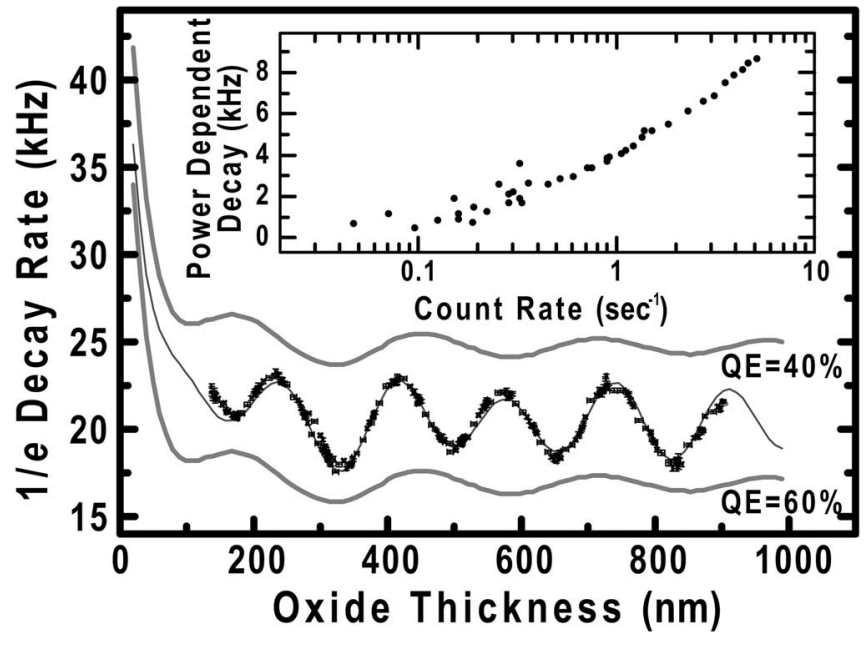

FIG. 2. Variation of the $750 \mathrm{~nm}$ Kohlrausch $1 / e$ decay rate $\Gamma_{K}$ with oxide thickness under constant, high power pump conditions (symbols). The periodicity in the data is explained by additional decay proportional to the local pump power (solid line). The grey drawn lines show bounding quantum efficiencies of $40 \%$ and $60 \%$ (see text). The inset shows the pump-power dependent decay component.

Figures 2 and 3(a) show $\Gamma_{K}$ measured at $750 \pm 5 \mathrm{~nm}$ for high and low pump powers, respectively. Both data sets show periodic variation in the decay rate as function of the oxide layer thickness. At high pump power (Fig. 2, symbols), the dominant variations match the periodicity expected for the interference pattern of the normal incidence $488 \mathrm{~nm}$ pump beam inside the oxide film (index of refraction $n$ $=1.48$ ). The solid line through the data is obtained by adding a decay component, that is proportional to the calculated local pump intensity inside the $\mathrm{SiO}_{2}$ film, to the low power data from Fig. 3(a). This interference pattern confirms the oxide thicknesses measured by spectral ellipsometry and the best-fit phase shift $(9 \pm 3 \mathrm{~nm})$ provides a verification of the assumed nanocrystal implantation depth.

It is apparent that $\Gamma_{K}$ depends on the pump intensity. The inset of Fig. 2 shows the residual power dependent decay component as function of the measured count rate (a measure of the local pump power). The origins of this additional decay component are not well understood. An earlier report of pump power dependent PL decay rate in sparse Si nanocrystal ensembles proposed a model of biexciton formation followed by Auger recombination. ${ }^{17}$ We speculate that internanocrystal nonradiative decay processes may also be important in dense ensembles. ${ }^{19}$ Photoionization associated with PL intermittence ("blinking") may also contribute. ${ }^{20}$

At low excitation powers the decay rate is almost independent of pump power and a variation that depends on emission wavelength dominates. The interference pattern of the $488 \mathrm{~nm}$ pump beam makes constant-power illumination an experimentally inefficient approach to maintaining low power excitation conditions. Instead, a feedback system was implemented on the pump laser intensity to maintain a roughly constant detection rate below 0.25 peak counts/s. The measured variation in the decay rate $\Gamma$ is well described by 

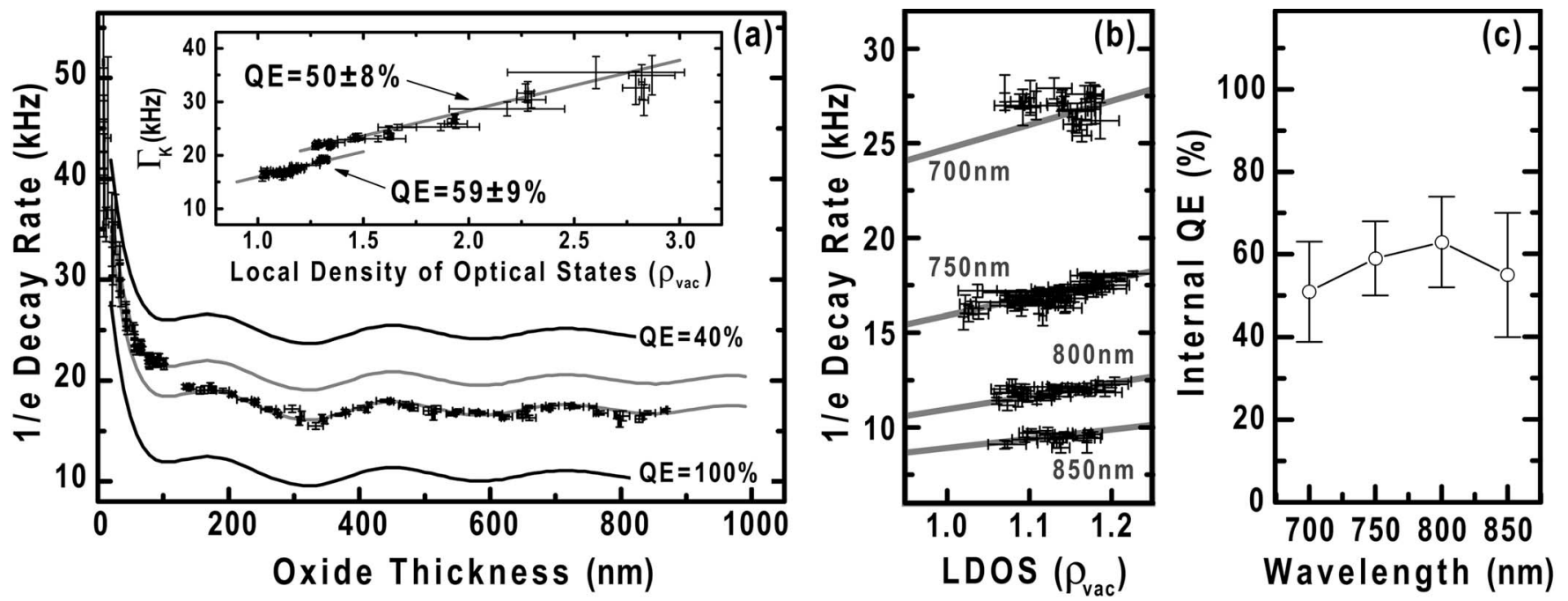

FIG. 3. (a) The Kohlrausch 1/e decay rate of silicon nanocrystals at $750 \mathrm{~nm}$ varies with oxide thickness in good agreement with a calculation of the local density of optical states. The ensemble radiative decay rate $(9.4 \pm 1.3 \mathrm{kHz})$ and quantum efficiencies for two samples are determined by a least squares fit to the linearized data (inset). (b) Measured total decay rate as function of LDOS at different wavelengths. (c) Quantum efficiency as function of wavelength suggesting that there is an optimal wavelength (nanocrystal size) for PL quantum efficiency.

$$
\Gamma=\Gamma_{\text {rad }} \rho(\omega, z)+\Gamma_{\text {nonrad }},
$$

where $\rho(\omega, z)$ is the LDOS at the position $z$ of the nanocrystal ensemble, and $\omega$ is the emission frequency. Equation (1) holds for both the measured Kohlrausch decay rate $\Gamma_{K}$ as well the intrinsic single-exponential decay rate $\Gamma_{0}$ because the relative change in $\Gamma_{K}$ is equal to the relative change in $\Gamma_{0}$. The non-radiative decay rate $\Gamma_{\text {nonrad }}$ is thought to depend primarily on the passivation quality at the nanocrystal-tooxide interface, independent of oxide thickness. This microscopic interfacial environment could vary from sample to sample (e.g., with changes in oxide quality or fabrication process) leading to differences in the intrinsic radiative rate and/or quantum efficiency.

To calculate the $\operatorname{LDOS} \rho(\omega, z)$ that describes the position dependent spontaneous emission rate we employ Fermi's Golden Rule to describe the coupling between the nanocrystal and the radiation field. ${ }^{7,10,21}$ We use $|i\rangle=|b\rangle \otimes\left|E_{i}\right\rangle$ and $|f\rangle=|a\rangle \otimes\left|E_{f}\right\rangle$ with energies $\hbar \omega_{i, f}$ as the initial and final states of the combined nanocrystal-radiation system of a nanocrystal with an excited state $|b\rangle$ and ground state $|a\rangle$. For the energy range of interest, the nanocrystal emission originates from recombination of quantum confined excitons in the dipole allowed singlet state. ${ }^{3,22}$ We therefore apply the electrical dipole approximation interaction Hamiltonian given by $\hat{H}_{\text {int }}=-\hat{\mu} \cdot \hat{E}(\vec{r})$ where $\hat{\mu}$ is the dipole operator and $\hat{E}(\vec{r})$ is the electrical field operator at the position of the nanocrystal. The decay rate can then be written as

$$
\begin{aligned}
\Gamma(\vec{r}) & =D_{a b}^{2} \sum_{\{n\}}\left\langle 0\left|\hat{E}(\vec{r})^{\dagger}\right| n\right\rangle\langle n|\hat{E}(\vec{r})| 0\rangle \delta\left(\omega-\omega_{n}\right) \\
& \equiv D_{a b}^{2} \frac{\hbar \omega}{2} \rho(\omega, \vec{r})
\end{aligned}
$$

where $D_{a b}^{2}=\frac{2 \pi}{\hbar^{2}}|\langle a|\hat{\mu}| b\rangle|^{2}$ is the oscillator strength for the tran- sition. The quantity $\rho(\omega, \vec{r})$ is the radiative LDOS and is calculated by quantizing the radiation field according to

$$
\hat{E}(\vec{r})=\sum_{\{j\}}\left[\frac{\hbar \omega_{j}}{2 \varepsilon(\vec{r})} i a_{j}^{\dagger} \varphi_{j}(\vec{r})+\text { H.c. }\right],
$$

where $a_{j}^{\dagger}$ is the creation operator of the mode $\left|\varphi_{j}(\vec{r})\right\rangle{ }^{10,21}$ The calculation of $\rho(\omega, \vec{r})$ sums a complete set of normalized plane waves at a single frequency for each polarization. The radiative mode functions $\varphi_{j}(\vec{r})$ contain both a propagating plane wave and a standing wave component dictated by the Fresnel coefficients of the two interfaces and the $\mathrm{SiO}_{2}$ film thickness. Guided modes are absent in our case as the refractive index of the $\mathrm{SiO}_{2}$ layer is lower than that of the $\mathrm{Si}$ substrate.

We use an isotropic combination of dipole orientations to calculate the LDOS at a constant distance of $10 \mathrm{~nm}$ from the $\mathrm{SiO}_{2}$-to-air interface corresponding to the position of the nanocrystals. This is justified as the PL is unpolarized and both the luminescence and measured decay are independent of the pump beam polarization. The result of this calculation is shown in Fig. 3(a) for different values of the internal quantum efficiency $\mathrm{QE}=\Gamma_{\text {rad }} /\left(\Gamma_{\text {rad }}+\Gamma_{\text {nonrad }}\right)$. The curves resemble those of earlier work with atoms close to a metal mirror. ${ }^{23}$ This is not surprising as the substrate reflects light efficiently at nanocrystal emission wavelengths. In our calculation we have neglected the effects of absorption and homogeneous broadening. A classical calculation of the loss rate of a dipole antenna ${ }^{7}$ that includes absorption differs from the above analysis by less than $2 \%$ at distances $<10 \mathrm{~nm}$ from the Si while perfect agreement is found at larger distances. Homogeneous broadening can be accounted for by averaging the calculated LDOS over a range of transition frequencies. This leads to a stronger damping of the modulation amplitude in the calculated emission rate as function 
of oxide thickness. For oxide thinner than one micron and a typical linewidth of order $100 \mathrm{meV}$ at room temperature ${ }^{4,24}$ the effect was found to be negligibly small.

The grey lines through the data in Fig. 3(a) correspond to a best fit of Eq. (1) using the LDOS calculated as a function of the total oxide thickness. The best fit assumes a common vacuum $(\mathrm{LDOS}=1.0)$ radiative emission rate $\Gamma_{\text {rad }}$ of $9.4 \pm 1.3 \mathrm{kHz}$ corresponding to quantum efficiencies of $59 \% \pm 9 \%$ and $50 \% \pm 8 \%$ for samples $A$ and $B$, respectively. ${ }^{25}$ To clearly show the linearity of the LDOS dependence, the data are also plotted against a linearized ordinate axis [inset of Fig. 3(a)]. We attribute the difference in quantum efficiency between samples $A$ and $B$ to sampleto-sample variation in the nonradiative decay rate. For comparison, the calculated total decay rates for quantum efficiency values of $40 \%$ and $100 \%$ are shown (black lines). The best fit radiative rate $\Gamma_{\text {rad }}$ implies an intrinsic radiative rate $\Gamma_{0}$ of $4.5 \pm 0.6 \mathrm{kHz}$ for an isolated Si nanocrystal emitting at $750 \mathrm{~nm}$ in an $(n=1.45)$ oxide matrix assuming that tunneling based energy transfer is responsible for the nonexponential decay. This value is in good agreement with total decay rate values reported $(6.5 \mathrm{kHz})$ for sparse nanocrystal ensembles of unknown quantum efficiency. ${ }^{17}$

The experiment can be repeated at other wavelengths to measure variation in both the intrinsic spontaneous emission rate and the quantum efficiency with nanocrystal size. However, the measured signal level decreases substantially away from $750 \mathrm{~nm}$ due to decreasing detector sensitivity and nanocrystal PL yield. Doubling the integration time to $2 \mathrm{~h}$ enabled measurements of the total decay rate over a limited range of LDOS values at other wavelengths. These data are plotted in Fig. 3(b). In order to determine quantum efficiency values from these measurements we assume that the intrinsic radiative rate of silicon nanocrystals varies according to an effective mass model appropriate to the size regime of the nanocrystals in this experiment. ${ }^{26}$ This allows us to scale $\Gamma_{\text {rad }}$ as measured at $750 \mathrm{~nm}$ to other wavelengths while determining the corresponding $\Gamma_{\text {nonrad }}$ experimentally. The results of this calculation are shown in Fig. 3(c), suggesting the existence of an optimum in PL quantum efficiency for nanocrystals that emit near $800 \mathrm{~nm} .{ }^{27}$ Note that the quantum efficiency values presented in this report pertain only to the optically active fraction of nanocrystals in the ensemble that contribute to the photoluminescence signal. The reported values should not be interpreted as an upper bound of what may be achievable in silicon nanocrystals. It is likely that improved fabrication methods will lead to even higher internal quantum efficiencies.

This work was supported by the Air Force Office of Scientific Research (MURI No. FA9550-04-1-0434) and the research program of FOM, which is financially supported by NWO. Technical development by Nicholas Chiang is gratefully acknowledged.
${ }^{1}$ L. T. Canham, Appl. Phys. Lett. 57, 1046 (1990).

${ }^{2}$ Silicon Photonics, Topics in Applied Physics Series Vol. 94, edited by L. Pavesi and D. J. Lockwood (Springer, New York, 2004).

${ }^{3}$ M. V. Wolkin, J. Jorne, P. M. Fauchet, G. Allan, and C. Delerue, Phys. Rev. Lett. 82, 197 (1999).

${ }^{4}$ J. Valenta, R. Juhasz, and J. Linnros, Appl. Phys. Lett. 80, 1070 (2002).

${ }^{5}$ G. M. Credo, M. D. Mason, and S. K. Buratto, Appl. Phys. Lett. 74, 1978 (1999).

${ }^{6}$ R. J. Walters, G. I. Bourianoff, and H. A. Atwater, Nat. Mater. 4, 143 (2005).

${ }^{7}$ W. L. Barnes, J. Mod. Opt. 45, 661 (1998).

${ }^{8}$ E. Snoeks, A. Lagendijk, and A. Polman, Phys. Rev. Lett. 74, 2459 (1995).

${ }^{9}$ M. J. A. de Dood et al., Phys. Rev. A 64, 033807 (2001).

${ }^{10}$ H. P. Urbach and G. L. J. A. Rikken, Phys. Rev. A 57, 3913 (1998).

${ }^{11}$ B. C. Buchler, T. Kalkbrenner, C. Hettich, and V. Sandoghdar, Phys. Rev. Lett. 95, 063003 (2005).

${ }^{12}$ J. Kalkman, H. Gersen, L. Kuipers, and A. Polman, Phys. Rev. B 73, 075317 (2006).

${ }^{13} \mathrm{http}: / /$ www.srim.org/.

${ }^{14}$ T. Feng, H. B. Yu, M. Dicken, J. R. Heath, and H. A. Atwater, Appl. Phys. Lett. 86, 33103 (2005).

${ }^{15}$ R. S. Anderssen, S. A. Husain and R. J. Loy, ANZIAM J. 45,
C800 (2004).

${ }^{16}$ M. L. Brongersma, A. Polman, K. S. Min, E. Boer, T. Tambo, and H. A. Atwater, Appl. Phys. Lett. 72, 2577 (1998).

${ }^{17}$ V. Vinciguerra, G. Franzo, F. Priolo, F. Iacona, and C. Spinella, J. Appl. Phys. 87, 8165 (2000).

${ }^{18}$ M. Inokuti and F. Hirayama, J. Chem. Phys. 43, 1978 (1965).

${ }^{19}$ D. Kovalev, H. Heckler, M. Ben-Chorin, G. Polisski, M. Schwartzkopff, and F. Koch, J. Porous Mater. 7, 85 (2000).

${ }^{20}$ F. Cichos, J. Martin, and C. von Borczyskowski, Phys. Rev. B 70, 115314 (2004).

${ }^{21}$ R. Sprik, B. A. van Tiggelen, and A. Lagendijk, Europhys. Lett. 35, 265 (1996).

${ }^{22}$ P. D. J. Calcott, K. J. Nash, L. T. Canham, M. J. Kane, and D. Brumhead, J. Phys.: Condens. Matter 5, L91 (1993).

${ }^{23}$ K. H. Drexhage, Prog. Opt. 12, 165 (1974).

${ }^{24}$ M. D. Mason, G. M. Credo, K. D. Weston, and S. K. Buratto, Phys. Rev. Lett. 80, 5405 (1998).

${ }^{25}$ If the vacuum $1 / e$ rate is allowed to vary independently for samples $A$ and $B$, best fit values of $10.7 \pm 0.4 \mathrm{kHz}$ and $7.5 \pm 0.4 \mathrm{kHz}$ are found. In this case the corresponding quantum efficiencies are $38 \% \pm 3 \%$ and $68 \% \pm 4 \%$.

${ }^{26}$ G. W. 't Hooft, Y. A. R. R. Kessener, G. L. J. A. Rikken, and A. H. J. Venhuizen, Appl. Phys. Lett. 61, 2344 (1992).

${ }^{27}$ M. Kapoor, K. Singh, and R. K. Pandey, Physica E (Amsterdam) 23, 183 (2004). 